\title{
TUG1 knockdown promoted viability and inhibited apoptosis and cartilage ECM degradation in chondrocytes via the miR-17-5p/FUT1 pathway in osteoarthritis
}

\author{
ZHICHAO LI ${ }^{1}$, JIN WANG ${ }^{1}$ and JING YANG ${ }^{2}$ \\ ${ }^{1}$ Department of Hand, Foot and Vascular Surgery, Hanyang Hospital, \\ Wuhan University of Science and Technology, Wuhan, Hubei 430050; \\ ${ }^{2}$ Department of Cardiology, Renmin Hospital, Wuhan University, \\ Wuhan, Hubei 430060, P.R. China
}

Received October 14, 2019; Accepted July 14, 2020

DOI: $10.3892 /$ etm.2020.9283

\begin{abstract}
Osteoarthritis (OA) is a degenerative disease characterized by cartilage destruction. Previous research has demonstrated that long non-coding RNAs serve a role in OA progression. The current study aimed to determine the function and mechanism of taurine upregulated gene (TUG) 1 in OA. The results of reverse transcription quantitative PCR revealed that TUG1 was elevated in OA cartilage tissues and interleukin (IL)-1 $\beta$-induced chondrocytes. Cell Counting kit-8 and flow cytometry analysis revealed that TUG1 knockdown promoted cell viability and inhibited cell apoptosis. Furthermore, matrix metalloprotein (MMP) 13, collagen II and aggrecan expression was determined by western blotting, of which the results demonstrated that TUG1 knockdown significantly decreased MMP13 expression and increased collagen II and aggrecan expression in IL-1 $\beta$-stimulated chondrocytes, indicating that extracellular matrix (ECM) damage was inhibited. Additionally, using bioinformatics analysis, dual-luciferase reporter and RNA immunoprecipitation assays, TUG1 was revealed to upregulate fucosyltransferase (FUT) 1 by targeting miR-17-5p. Furthermore, miR-17-5p was downregulated and FUT1 upregulated in OA cartilage tissues and IL-1 $\beta$-induced chondrocytes. TUG1 overexpression reversed the aforementioned effects on cell viability, cell apoptosis and ECM degradation mediated by miR-17-5p in IL-1 $\beta$-activated chondrocytes. Additionally, the effects of FUT1 knockdown on cell viability, apoptosis and ECM degradation mediated by
\end{abstract}

Correspondence to: Mr. Jing Yang, Department of Cardiology, Renmin Hospital, Wuhan University, 238 Jiefang Road, Wuhan, Hubei 430060, P.R. China

E-mail: liman28194@163.com

Key words: osteoarthritis, taurine upregulated gene 1 , microRNA-17-5p, fucosyltransferase 1, extracellular matrix
FUT1 knockdown were reversed by miR-17-5p inhibition. In conclusion, TUG1 knockdown inhibited OA progression by downregulating FUT1 via miR-17-5p.

\section{Introduction}

Osteoarthritis (OA) is a degenerative joint disease characterized by articular cartilage degeneration and a high incidence rate in the elderly population $(1,2)$. Chondrocyte degradation and synthesis imbalance, extracellular matrix (ECM) and subchondral bones are involved in the degradation of articular cartilage, which leads to OA progression $(3,4)$. $\mathrm{OA}$ is a disease that affects the quality of life in the elderly worldwide (5). However, the pathogenesis of OA has not been fully elucidated. Thus, exploring the pathogenesis of OA is necessary.

Long non-coding RNAs (lncRNAs) are non-coding RNAs (ncRNAs) $>200$ nucleotides (nts) in length that serve crucial roles in various biological processes, such as tumorigenesis, cell growth, metastasis and apoptosis $(6,7)$. lncRNA taurine upregulated gene 1 (TUG1) has been reported to be highly expressed and involved in the development of human diseases. For example, Niu et al (8) demonstrated that TUG1 was upregulated in small cell lung cancer (SCLC), that TUG1 depletion inhibited SCLC cell growth and metastasis, and that TUG1 induced apoptosis. Huang et al (9) reported increased TUG1 levels in hepatocellular carcinoma (HCC) and demonstrated that TUG1 inhibition repressed HCC progression. While various lncRNAs, such as HOX transcript antisense RNA (10), maternally expressed gene 3 (11) and X-inactive specific transcript (12) have been demonstrated to participate in OA regulation, the association between TUG1 and OA remains unclear. Thus, the current study aimed to investigate the functional role and potential mechanism of TUG1 in the progression of OA.

MicroRNAs (miRNAs or miRs) are a series of ncRNAs 19-25 nts in length that modulate gene expression by recognizing the 3 '-untranslated region (3'-UTR) of target 
genes (13). Numerous studies have reported that miRs are dysregulated in OA and serve various roles in OA development. For instance, Huang et al (14) demonstrated that miR-204 and miR-211 maintained joint homeostasis and inhibited OA progression. Meng et al (15) reported decreased miR-193b-3p expression in OA, while miR-193b-3p upregulation promoted cartilage formation by interacting with histone deacetylase 3. However, Cai et al (16) determined that miR-27a expression was elevated in OA cartilage tissues and interleukin (IL)-1 $\beta$-induced chondrocytes and facilitated chondrocyte apoptosis. Furthermore, Yang et al (17) reported elevated miR-145 expression in IL-1 $\beta$-stimulated chondrocytes, leading to ECM degradation in OA cartilage. These data suggested that miRNAs served different roles in OA development.

A previous study reported that miR-17-5p was associated with OA (10). However, the specific pathogenesis of miR-17-5p in OA has not been fully elucidated. As lncRNAs have been reported to alter mRNA expression as mRNA targets (18), further research is required to establish whether TUG1 targets miR-27-5p.

Fucosyltransferases (FUTs) are a group of glycosylation synthetases (19). Previous studies have revealed that certain FUT genes may serve important roles in OA. For example, FUT4 was abnormally expressed and reportedly involved in IL-1 $\beta$-mediated ECM regulation, chondrocyte growth and apoptosis in OA (20). FUT2 expression was also reportedly elevated in OA and aggravated OA progression by impairing the ECM, promoting cell apoptosis and inhibiting cell growth (10). To the best of our knowledge, there are few reports regarding FUT1 in OA and few have assessed whether miR-17-5p targets FUT1.

In the current study, TUG1, miR-17-5p and FUT1 expression was determined in OA and their role and mechanism of action in the regulation of ECM degradation, cell viability and apoptosis.

\section{Materials and methods}

Tissue collection. The current study was approved by the Ethics Committee of Hanyang Hospital affiliated with Wuhan University of Science and Technology. Written informed consent was obtained from all participants.

A total of 25 OA cartilage tissue samples (10 male and 15 female; age, 52-70 years) were collected from patients who had undergone knee or hip arthroplasty and 25 normal cartilage tissue samples (12 male and 13 female; age, 35-46 years) were obtained from patients who underwent traumatic amputations at Hanyang Hospital between October 2016 and March 2018. OA patients were diagnosed according to the American College of Rheumatology criteria (21). The control individuals had no history of joint disease. At the time of surgery, the patients had symptomatic disease requiring medical treatment. None had received intra-articular steroid injections within 3 months prior to surgery. Samples were stored at $-80^{\circ} \mathrm{C}$ for total RNA and protein extraction.

Cell culture. Chondrocytes were collected from OA cartilage tissues and cut into small sections $\left(<1 \mathrm{~mm}^{3}\right)$. Samples were pre-treated with $0.25 \%$ trypsin (Beijing Solarbio Science
\& Technology Co., Ltd.) for $10 \mathrm{~min}$ and then digested with $0.2 \%$ collagenase II (Beijing Solarbio Science \& Technology Co., Ltd.) in DMEM (Nissui Pharmaceutical, Co., Ltd.) supplemented with 10\% FBS (Beijing Solarbio Science \& Technology Co., Ltd.) overnight at $37^{\circ} \mathrm{C}$. Undigested samples were removed with a filter $(40 \mu \mathrm{m}$; Beijing Solarbio Science \& Technology Co., Ltd.). Chondrocytes were collected following centrifugation at $2,000 \mathrm{xg}$ for $5 \mathrm{~min}$ at $37^{\circ} \mathrm{C}$.

All chondrocytes were cultured in DMEM (Nissui Pharmaceutical, Co., Ltd.) supplemented with 10\% FBS (Beijing Solarbio Science \& Technology Co., Ltd.) and $1 \%$ penicillin-streptomycin (Beijing Solarbio Science \& Technology Co., Ltd.) at $37^{\circ} \mathrm{C}$ in a humidified atmosphere with $5 \% \mathrm{CO}_{2}$. Chondrocytes at passages 2 and 3 were analyzed.

Cell transfection and IL-1 $\beta$ treatment. Small interfering RNA (siRNA) targeting TUG1 (si-TUG1; 5'-CCAUCUCAC AAGGCUUCAATT-3'), FUT1 (si-FUT1; 5'-UCGAUGUUU UCUUUACACCAC-3') and controls (si-NC; 5'-UUCUCC GAACGUGUCACGUTT-3'); the pcDNA3.1-TUG1 overexpression vector (pc-TUG1) and corresponding empty vector (vector); miR-17-5p mimics (miR-17-5p; 5'-CAAAGUGCU UACAGUGCAGGUAG-3') and controls (miR-NC; 5'-UUC UCCGAACGUGUCACGUTT-3'); and miR-17-5p inhibitors (anti-miR-17-5p; 5'-CAAAGUGCUUACAGUGCAGGU AG-3') and controls (anti-miR-NC; 5'-CAGUACUUUUGU GUAGUACAA-3') were purchased from GeneCopoeia, Inc. Chondrocytes were seeded into 24 -well plates at a density of $1.0 \times 10^{4}$ cells/well and the oligonucleotides $(50 \mathrm{nM})$ or vectors $(2 \mu \mathrm{g})$ were transfected into chondrocytes using Lipofectamine $^{\circledast} 2000$ (Invitrogen; Thermo Fisher Scientific, Inc.).

Chondrocytes were stimulated with $10 \mathrm{ng} / \mathrm{ml} \mathrm{IL-1 \beta}$ (Beyotime Institute of Biotechnology) for $24 \mathrm{~h}$ at $37^{\circ} \mathrm{C}$. Untreated normal primary chondrocytes were used as controls.

Reverse transcription-quantitative PCR (RT-qPCR). Cartilage tissue samples and chondrocytes were lysed with RNAiso Plus (Takara Biotechnology Co., Ltd.) to extract total RNA and concentrations were measured using a NanoDrop 2000c spectrophotometer (Thermo Fisher Scientific, Inc.). RNA was reverse transcribed into cDNA using the HiScript ${ }^{\circledR}$ II Reverse Transcriptase kit (Vazyme Biotech Co., Ltd.) under the thermal conditions of $50^{\circ} \mathrm{C}$ for $15 \mathrm{~min}$ followed by $85^{\circ} \mathrm{C}$ for $5 \mathrm{sec}$ or miRNA using the 1st Strand cDNA Synthesis kit (Vazyme Biotech Co., Ltd.) under the thermal conditions of $25^{\circ} \mathrm{C}$ for $5 \mathrm{~min}, 50^{\circ} \mathrm{C}$ for $15 \mathrm{~min}$ followed by $85^{\circ} \mathrm{C}$ for $5 \mathrm{~min}$. RT-qPCR was performed using a AceQ Universal SYBR qPCR Master Mix (Vazyme Biotech Co., Ltd.) on an ABI 7500 PCR system (Applied Biosystems; Thermo Fisher Scientific, Inc.). The thermocycling conditions of the qPCR reaction were: i) Initial denaturation at $95^{\circ} \mathrm{C}$ for $5 \mathrm{~min}$; ii) 40 cycles of $95^{\circ} \mathrm{C}$ for $10 \mathrm{sec}$ and $60^{\circ} \mathrm{C}$ for $30 \mathrm{sec}$; iii) $95^{\circ} \mathrm{C}$ for $15 \mathrm{sec}, 60^{\circ} \mathrm{C}$ for $60 \mathrm{sec}$ and $95^{\circ} \mathrm{C}$ for $15 \mathrm{sec}$. TUG1, FUT1 and miR-17-5p expression was calculated using the $2^{-\Delta \Delta \mathrm{Cq}}$ method (22) with GAPDH or U6 as controls. The primers were as follows: TUG1 forward, 5'-TAG CAGTTCCCCAATCCTTG-3' and reverse, 5'-CACAAATTC CCATCATTCCC-3'; miR-17-5p forward, 5'-TGCGCCAAA GTGCTTACAGTGCA-3' and reverse, 5'-CCAGTGCAGGGT 
CCGAGGTATT-3'; FUT1 forward, 5'-AAAGCGGACTGT GGATCT-3' and reverse, 5'-GGACACAGGATCGACAGG-3'; GAPDH forward, 5'-TGTTCGTCATGGGTGTGAAC-3' and reverse, 5'-ATGGCATGGACTGTGGTCAT-3'; U6 forward, 5'-CTTCGGCAGCACATATACT-3' and reverse, 5'-AAAATA TGGAACGCTTCACG-3'.

Cell counting kit-8 (CCK-8) assay. Chondrocyte viability was assessed using a CCK-8 assay following transfection and treatment. Collected chondrocytes $\left(5.0 \times 10^{3}\right.$ cells/well) were seeded into 96-well plates and $10 \mu \mathrm{l}$ CCK- 8 reagent (Beyotime Institute of Biotechnology) was added to each well at $48 \mathrm{~h}$. After incubation for $4 \mathrm{~h}$ at $37^{\circ} \mathrm{C}$ and $5 \% \mathrm{CO}_{2}$, absorbance at $450 \mathrm{~nm}$ was determined using a microplate reader (BioTek Instruments, Inc.).

Flow cytometry analysis. Chondrocyte apoptosis was evaluated using an Annexin FITC/PI Apoptosis Detection kit (Beyotime Institute of Biotechnology) according to the manufacturer's instructions following transfection and treatment. Chondrocytes were harvested, washed with PBS (Beijing Solarbio Science \& Technology Co., Ltd.), resuspended at a concentration of $1.0 \times 10^{6}$ cells $/ \mathrm{ml}$ and incubated with $5 \mu \mathrm{l}$ Annexin V-FITC and $10 \mu \mathrm{l}$ PI for $10 \mathrm{~min}$ at room temperature in the dark. Chondrocyte apoptotic rate was analyzed using a FACScan ${ }^{\circledast}$ flow cytometer (BD Biosciences) for $1 \mathrm{~h}$. The level of apoptotic cells were analyzed using FlowJo 7.6.1. (FlowJo LLC). Apoptosis rate was calculated as the sum of the early apoptosis rate (the lower right quadrant) and the late apoptosis rate (the upper right quadrant).

Western blot assay. Total protein was extracted by lysing cartilage tissues and chondrocytes with RIPA buffer (Beyotime Institute of Biotechnology) and quantified using a NanoDrop 2000c spectrophotometer (Thermo Fisher Scientific, Inc.). Protein samples (30 $\mu$ g/lane) were subjected to $10 \%$ SDS gel (Beijing Solarbio Science \& Technology Co., Ltd.) and transferred onto polyvinylidene difluoride membranes (Pall Life Sciences). Membranes were blocked with 5\% non-fat milk for $1 \mathrm{~h}$ at room temperature and then incubated with primary antibodies against FUT1 (cat. no. bs-7636R; BIOSS; 1:200), matrix metalloprotein 13 (MMP13; cat. no. bs-10250R; BIOSS; 1:300), collagen II (cat. no. bs-11929R; BIOSS; 1:1,000), aggrecan (cat. no. ab3778; Abcam; 1:100) or GAPDH (cat. no, ab9485; Abcam; 1:2,000) overnight at $4^{\circ} \mathrm{C}$. Samples were then incubated with horseradish peroxidase-conjugated secondary antibodies (cat. no. bs-40296G; BIOSS; 1:5,000) for $1 \mathrm{~h}$ at room temperature. Bands were detected using an enhanced chemiluminescence kit (Beyotime Institute of Biotechnology) according to the instructions of manufacturer and analyzed using ImageJ v1.8.0 (National Institutes of Health).

Dual-luciferase reporter assay. Potential binding sites between TUG1 and miR-17-5p, and miR-17-5p and FUT1 were predicted by online software MIRcode 11 (http://www.mircode.org/) and starBase2.0 (http://starbase.sysu.edu.cn/index.php) and then verified using a dual-luciferase reporter assay. TUG1 and FUT1 wild type (WT) 3'-UTR sequences containing potential miR-17-5p binding sites and the sequences of TUG1 and FUT1 mutant (MUT) 3'-UTR, with the binding site removed, were cloned into a pGL3 vector (Promega Corporation) to generate TUG1 WT, FUT1 WT, TUG1 MUT and FUT1 MUT luciferase reporter vectors. Chondrocytes were then seeded into 24-well plates $\left(5.0 \times 10^{4}\right.$ cells/well) and co-transfected with indicated vectors (100 ng) and miR-17-5p or miR-NC (50 $\mathrm{nM})$ utilizing Lipofectamine ${ }^{\circledR} 2000$ (Invitrogen; Thermo Fisher Scientific, Inc.). Cells were gathered after $48 \mathrm{~h}$ and a dual-luciferase reporter assay kit (Promega Corporation) was used to detect luciferase activity according to the instructions of manufacturer. Renilla luciferase activity was normalized to firefly luciferase activity.

RNA immunoprecipitation (RIP) assay. An RIP assay was performed to determine whether TUG1 was expressed in the RNA-induced silencing complex using a Magna RNA-binding protein immunoprecipitation kit (EMD Millipore). Chondrocytes $\left(2.0 \times 10^{5}\right.$ cells) were collected, lysed in RIP buffer (Beijing Solarbio Science \& Technology Co., Ltd.) and incubated with magnetic beads (cat. no. 88847; Thermo Fisher Scientific, Inc.) conjugated with anti-argonaute-2 (anti-Ago2; cat. no. ab32381; Abcam; $2 \mu \mathrm{g} / \mathrm{ml}$ ) or anti-immunoglobulin $\mathrm{G}$ (IgG; cat. no. ab133470; Abcam; 1:5,000) overnight at $4^{\circ} \mathrm{C}$ following incubation with Proteinase K (Beijing Solarbio Science \& Technology Co., Ltd.) at $55^{\circ} \mathrm{C}$ for $30 \mathrm{~min}$. Purified RNA was isolated from the magnetic beads using RNAiso Plus (Takara Biotechnology Co., Ltd.) and analyzed via the aforementioned RT-qPCR assay to determine TUG1 and miR-17-5p enrichment.

Statistical analysis. Statistical analysis was performed using GraphPad Prism software (version 7; GraphPad Software, Inc.). All experiments were performed in triplicate and data were presented as the mean \pm standard deviation. Paired Student's t-test or one-way ANOVA followed by Tukey's test was used for analysis between groups. Spearman's correlation analysis was used to analyze the association between TUG1, miR-17-5p and FUT1 in OA cartilage tissue. $\mathrm{P}<0.05$ was considered to indicate a statistically significant difference.

\section{Results}

TUG1 is highly expressed in OA cartilage tissue and $I L-1 \beta$-induced chondrocytes. RT-qPCR was performed to analyze TUG1 expression in OA cartilage tissue and normal cartilage tissue (control) and to determine the potential role of TUG1 in the development of OA. The results demonstrated that TUG1 expression was significantly increased in OA cartilage tissue and IL- $1 \beta$-activated chondrocytes compared with controls (Fig. 1A and B). These data indicated that abnormal TUG1 expression may be involved in OA development.

TUG1 knockdown promotes IL-1 $\beta$-induced cell viability and inhibits cell apoptosis and ECM degradation in chondrocytes. Chondrocytes were transfected with si-TUG1 or si-NC and treated with IL- $1 \beta$ to investigate the functional role of TUG1 in cell viability, apoptosis and ECM degradation in OA. RT-qPCR demonstrated that IL- $1 \beta$-induced 

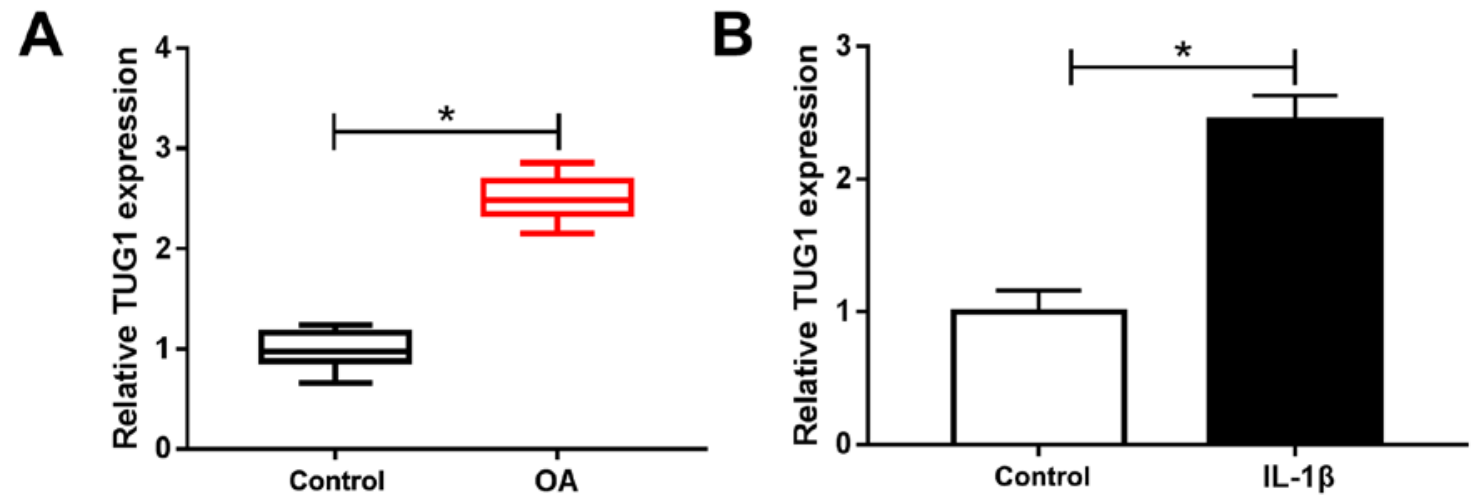

Figure 1. Elevated TUG1 expression in OA cartilage tissues and IL-1 $\beta$-induced chondrocytes. (A) TUG1 expression in OA cartilage tissues and controls was measured using RT-qPCR. (B) TUG1 expression in IL-1 $\beta$-activated chondrocytes and controls was also examined via RT-qPCR. "P<0.05 as indicated. TUG1, taurine upregulated gene 1; OA, osteoarthritis; IL, interleukin; control, normal primary chondrocytes; RT-qPCR; reverse transcription quantitative PCR.

TUG1 upregulation was markedly decreased in chondrocytes transfected with si-TUG1 compared with the si-NC transfected group (Fig. 2A). Chondrocyte viability was significantly inhibited and cell apoptosis was promoted by IL-1 $\beta$ treatment in comparison with control, with TUG1 knockdown partially reversing these effects, as demonstrated by the CCK-8 and flow cytometry assays, respectively (Fig. 2B and C). Furthermore, western blotting was performed to determine MMP13, collagen II and aggrecan expression. The results demonstrated that MMP13 expression was significantly increased following IL-1 $\beta$ treatment, while collagen II and aggrecan were significantly decreased, with TUG1 knockdown partially reversing these effects (Fig. 2D). These data suggested that IL-1 $\beta$ treatment decreased cell viability and promoted cell apoptosis and ECM degradation, and that TUG1 knockdown abrogated these effects.

TUG1 negatively modulates miR-17-5p expression via direct interaction in chondrocytes. MIRcode11 online software was used to determine potential TUG1 binding sites to investigate the underlying mechanism of TUG1 in OA. The results indicated that miR-17-5p was a predicted target of TUG1 (Fig. 3A) and that miR-17-5p transfection led to a significant increase in miR-17-5p expression, indicating that miR-17-5p was successfully transfected (Fig. 3B). Furthermore, the dual-luciferase reporter assay demonstrated that luciferase activity in TUG1 WT and miR-17-5p co-transfected chondrocytes was significantly decreased compared with TUG1 WT and miR-NC. Activity was unaltered in the TUG1 MUT group (Fig. 3C). The RIP assay indicated that TUG1 and miR-17-5p were significantly expressed in anti-Ago2 immunoprecipitates compared with anti-IgG (Fig. 3D).

Furthermore, miR-17-5p expression in OA cartilage tissues and IL-1 $\beta$-stimulated chondrocytes was assessed using RT-qPCR. It was revealed that miR-17-5p expression was significantly decreased in OA cartilage tissues and IL-1 $\beta$-stimulated chondrocytes compared with controls (Fig. E and F). miR-17-5p was also revealed to be negatively correlated with TUG1 in OA cartilage tissues, as demonstrated by Spearman's correlation analysis (Fig. 3G). Additionally, TUG1 knockdown resulted in significantly increased miR-17-5p expression, while TUG1 overexpression resulted in the significant inhibition of miR-17-5p expression in chondrocytes (Fig. $3 \mathrm{H}$ ). The results revealed that TUG1 directly targeted miR-17-5p and negatively modulated miR-17-5p expression in chondrocytes.

Upregulation of TUG1 attenuates the effects of miR-17-5p overexpression on cell viability, cell apoptosis and ECM degradation in IL-1 $\beta$-stimulated chondrocytes. Chondrocytes were untransfected or transfected with IL-1 $\beta$, IL- $1 \beta+$ miR-NC, IL- $1 \beta+$ miR-17-5p, IL- $\beta$ + miR-17-5p + vector or IL-1 $\beta+$ miR-17-5p + pc-TUG1 and miR-17-5p expression was determined to investigate whether TUG1 regulated cell viability, apoptosis and ECM degradation by targeting miR-17-5p in IL-1 $\beta$-stimulated chondrocytes. The results revealed that IL-1 $\beta$-mediated miR-17-5p downregulation was reversed by miR-17-5p transfection and that TUG1 overexpression inhibited this effect (Fig. 4A). IL-1 $\beta$-mediated cell viability suppression was significantly increased following miR-17-5p transfection as demonstrated by a CCK- 8 assay. However, this increase was partly abrogated by TUG1 overexpression (Fig. 4B). Flow cytometry analysis revealed that IL-1 $\beta$ increased cell apoptosis and that this was significantly decreased by miR-17-5p transfection. However, TUG1 overexpression reversed this effect (Fig. 4C). Western blotting demonstrated increased MMP13 and decreased collagen II and aggrecan expression following IL- $1 \beta$ treatment. These levels were subsequently abolished by miR-17-5p and restored by TUG1 overexpression (Fig. 4D). In summary, miR-17-5p overexpression reversed the effect of IL- $1 \beta$ on cell viability, apoptosis and ECM degradation, and these effects were reversed by TUG1.

miR-17-5p inhibits FUT1 expression in chondrocytes. The online software, starBase2.0, was used to identify FUT1 as a target gene of miR-17-5p. Their potential binding sites were established (Fig. 5A). si-FUT1 transfection resulted in significant reduction in FUT1 mRNA and protein levels (Fig. 5B and C). The dual-luciferase reporter assay revealed that FUT1 WT and miR-17-5p cotransfection resulted in a significant decrease in luciferase activity, while FUT1 MUT and miR-17-5p or miR-NC cotransfection had no effect (Fig. 5D). FUT1 mRNA and protein OA cartilage tissues 

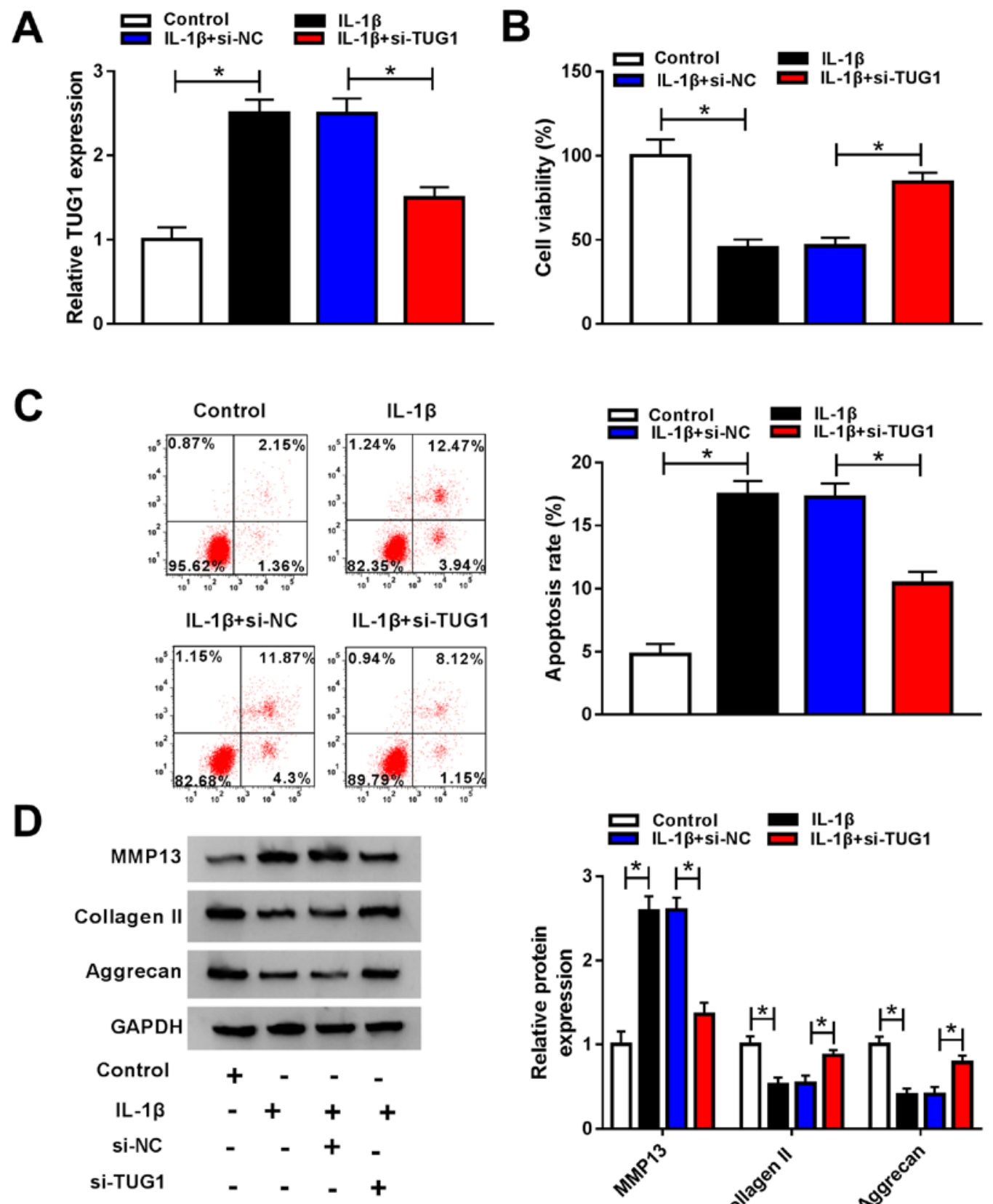

Figure 2. TUG1 knockdown reversed IL-1 $\beta$-induced effects on cell viability, cell apoptosis and extracellular matrix degradation in chondrocytes. Chondrocytes were untreated or treated with IL-1 $\beta$, IL-1 $\beta+$ si-NC or IL-1 $\beta+$ si-TUG1. (A) TUG1 expression was assessed via reverse transcription quantitative PCR. (B) Cell viability was evaluated using a Cell Counting kit 8 assay. (C) Apoptosis rate was analyzed via flow cytometry analysis. (D) MMP13, collagen II and aggrecan expression was determined via western blotting. "P $<0.05$ as indicated. TUG1, taurine upregulated gene 1; IL, interleukin; siRNA, small interfering RNA; si-NC, Small interfering RNA negative control; si-TUG1, small interfering RNA targeting TUG1; MMP13, matrix metalloprotein 13.

were significantly elevated compared with controls, as demonstrated by RT-qPCR and western blotting, respectively (Fig. 5E and F). Furthermore, the results revealed an inverse correlation between FUT1 and miR-17-5p in OA cartilage tissues (Fig. 5G).

FUT1 mRNA and protein levels of FUT1 were increased in IL-1 $\beta$-induced chondrocytes compared with controls (Fig. 5H and I). Furthermore, miR-17-5p inhibited FUT1 mRNA and protein expression and miR-17-5p knockdown had the opposite effect (Fig. 5J and K). These results revealed that miR-17-5p directly targeted FUT1 and inhibited FUT1 expression in chondrocytes.
miR-17-5p knockdown reverses the effects of $I L-1 \beta$ on cell viability, apoptosis and ECM degradation mediated by FUT1 knockdown in IL-1 $\beta$-stimulated chondrocytes. Since miR-17-5p directly regulated FUT1 expression in chondrocytes, the effect of miR-17-5p on chondrocyte viability, apoptosis and ECM degradation was investigated. Chondrocytes were untransfected or transfected with IL-1 $\beta$, IL-1 $\beta+$ si-NC, IL-1 $\beta+$ si-FUT1, IL- $\beta$ + si-FUT1 + anti-miR-NC or IL-1 $\beta+$ si-FUT1 + anti-miR-17-5p, after which FUT1 mRNA and protein levels were analyzed. IL-1 $\beta$ treatment caused a significant increase in FUT1 mRNA and protein levels. FUT1 knockdown significantly reduced these levels, which were 

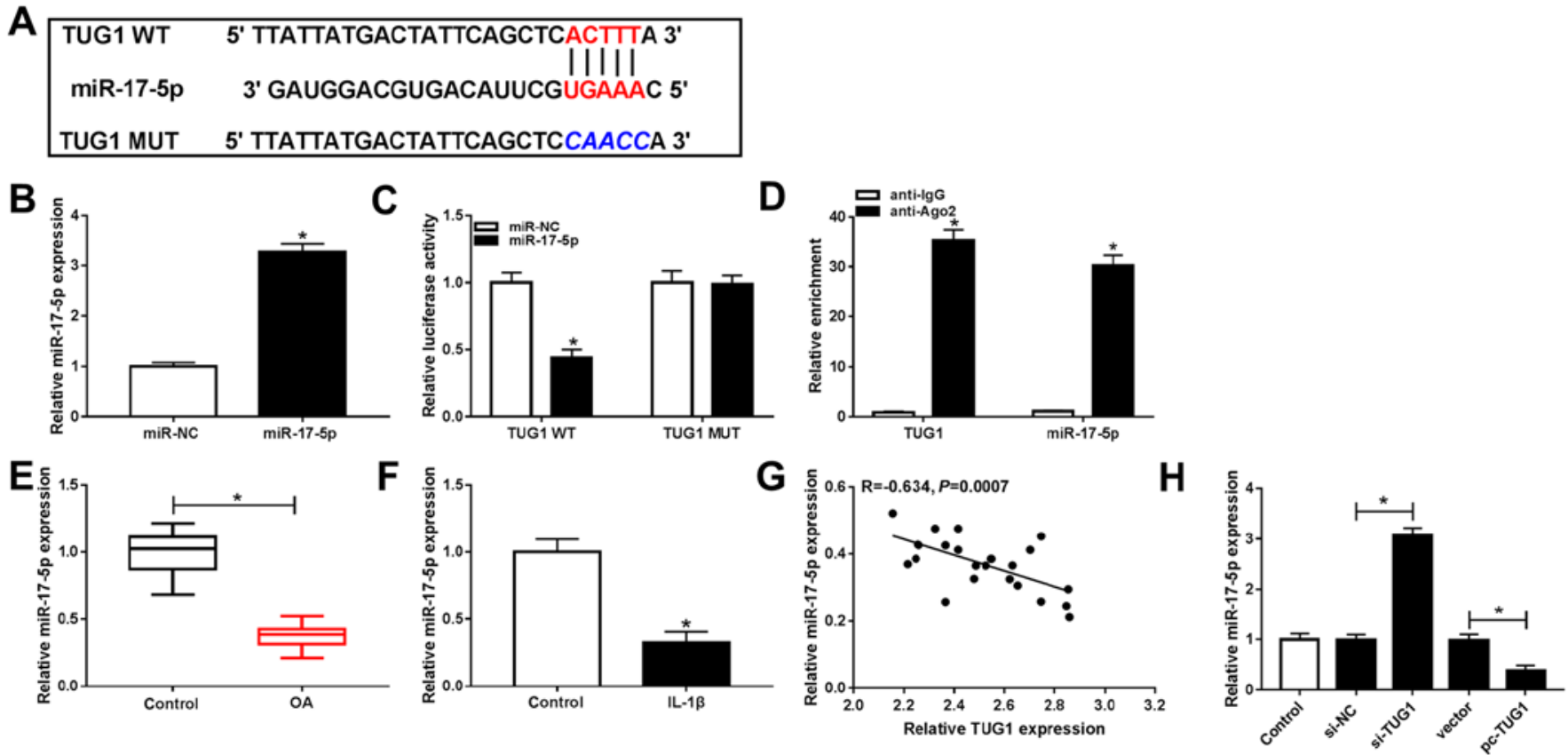

Figure 3. TUG1 negatively regulated miR-17-5p expression via direct targeting in chondrocytes. (A) Potential binding sites between TUG1 and miR-17-5p. (B) miR-17-5p expression following transfection with miR-17-5p or miR-NC was determined via RT-qPCR. (C) Luciferase activity in TUG1 WT or TUG1 MUT and miR-17-5p and miR-NC co-transfected chondrocytes was analyzed using a dual-luciferase reporter assay. (D) An RNA immunoprecipitation assay was performed to assess the association between TUG1 and miR-17-5p, and expressions were determined via RT-qPCR. (E) miR-17-5p expression in OA cartilage tissues and controls was measured via RT-qPCR. (F) miR-17-5p expression in IL-1 1 -stimulated chondrocytes and controls was examined via RT-qPCR. (G) Correlation between the expression of TUG1 and miR-17-5p in OA cartilage tissues was analyzed via Spearman's correlation analysis. (H) miR-17-5p expression in untransfected or transfected chondrocytes with si-NC, si-TUG1, vector or pc-TUG1 was determined via RT-qPCR. "P<0.05 vs. the miR-NC group, ${ }^{*} \mathrm{P}<0.05$ vs. the anti-IgG group, ${ }^{*} \mathrm{P}<0.05$ vs. the Control groups, ${ }^{*} \mathrm{P}<0.05$ vs. the si-NC groups, ${ }^{*} \mathrm{P}<0.05$ vs. the Vector groups. TUG1, taurine upregulated gene 1; miR, microRNA; miR-NC, microRNA negative control; RT-qPCR, reverse transcription quantitative-PCR; WT, wild type; MUT, mutant; OA, osteoarthritis; IL, interleukin; siRNA, small interfering RNA; si-NC, small interfering RNA negative control; si-TUG1, small interfering RNA targeting TUG1.

partially rescued by miR-17-5p inhibition (Fig. 6A and B). The CCK-8 assay revealed that FUT1 knockdown restored IL-1 $\beta$-mediated suppression in cells. This effect was reversed by miR-17-5p inhibition (Fig. 6C). IL-1 $\beta$ treatment also mediated apoptosis. FUT1 knockdown inhibited this apoptosis; however, this effect was significantly reversed following anti-miR-17-5p transfection (Fig. 6D). The results of western blotting revealed that increased levels of MMP13 and collagen II, and decreased levels of aggrecan were rescued following si-FUT1 transfection. However, this effect was reversed following anti-miR-17-5p transfection (Fig. 6E). The results demonstrated that miR-17-5p downregulation reversed the effects of FUT1 depletion on cell viability, apoptosis and ECM degradation in IL-1 $\beta$-induced chondrocytes.

TUG1 regulates FUT1 expression by targeting miR-17-5p in chondrocytes. si-NC, si-TUG1, si-TUG1 + anti-miR-NC or si-TUG1 + anti-miR-17-5p were transfected into chondrocytes with untransfected cells as controls to investigate the association between TUG1, miR-17-5p and FUT1. FUT1 mRNA and protein levels were significantly decreased following si-TUG1 transfection compared with si-NC. This was reversed by anti-miR-17-5p transfection (Fig. 7A and B). Furthermore, Spearman's correlation analysis revealed a positive correlation between FUT1 and TUG1 mRNA expression in OA cartilage tissues (Fig. 7C). These data indicated that TUG1 positively modulated FUT1 expression by targeting miR-17-5p in chondrocytes.

\section{Discussion}

Accumulating evidence has demonstrated that IncRNA dysregulation serves a vital role in the pathogenesis of OA $(23,24)$. In the current study, the function and mechanism of TUG1 in OA was analyzed. The results revealed that TUG1 and FUT1 were significantly expressed and that miR-17-5p was significantly inhibited. Additionally, TUG1 knockdown improved cell viability and inhibited cell apoptosis and ECM degradation via the miR-17-5p/FUT1 pathway in OA.

IL-1 $\beta$ impairs cartilage formation in the ECM, induces apoptosis and represses chondrocyte growth (25-27). In the present study, chondrocytes were exposed to IL-1 $\beta$ to mimic the OA microenvironment. The results revealed that cell viability was suppressed and cell apoptosis was induced in IL-1 $\beta$-stimulated chondrocytes. As cartilage ECM is mainly composed of collagen II and aggrecan, and MMP13 is a typical cartilage-degrading enzyme that serves a vital role in ECM degradation $(28,29)$, their levels were also evaluated. The results demonstrated increased MMP13 and decreased collagen II and aggrecan expression, indicating that ECM degradation was promoted.

The current study reported a significant elevation in TUG1 expression in OA cartilage tissues and IL-1 $\beta$-stimulated chondrocytes. Liang and Ren (30) revealed that emodin increased cell viability and inhibited cell apoptosis and inflammation via TUG1 elevation in LPS-stimulated ATDC5 cells. 
A
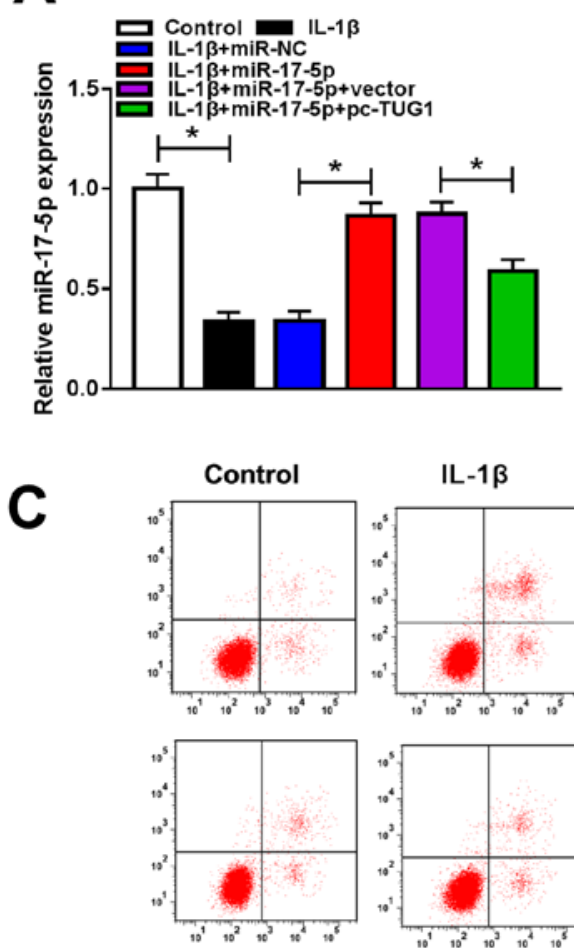

IL-1 $\beta+$ miR-17-5p

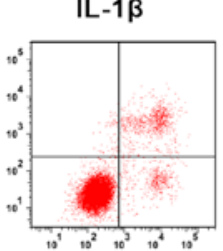

B

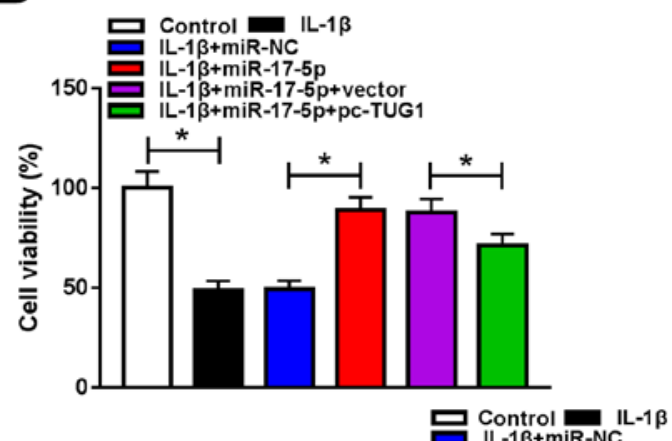

IL-1 $\beta+\operatorname{miR}-\mathrm{NC}$
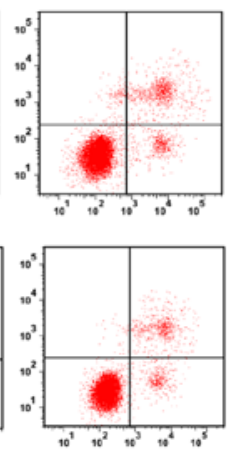

IL-1 $\beta+m i R-17-5 p$ + pc-TUG1

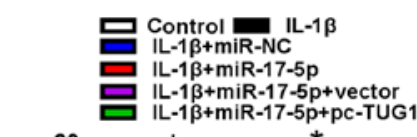

D
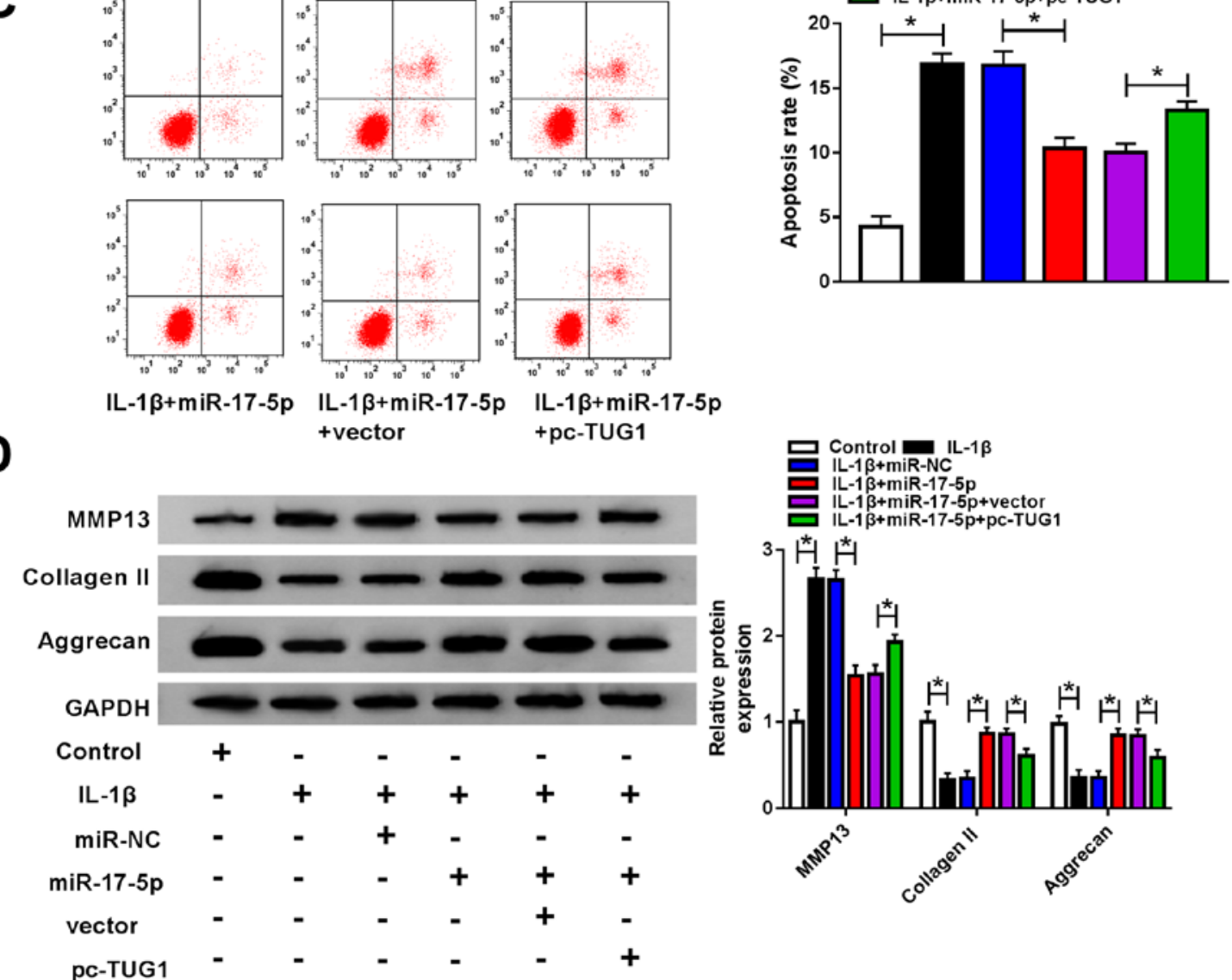

Figure 4. TUG1 overexpression reversed the effects of miR-17-5p overexpression on cell viability, apoptosis and extracellular matrix degradation in IL-1 $\beta$-activated chondrocytes. Chondrocytes were untransfected or transfected with IL-1 $\beta$, IL-1 $\beta+$ miR-NC, IL-1 $\beta+$ miR-17-5p, IL-1 $\beta+$ miR-17-5p + vector or IL-1 $\beta+$ miR-17-5p + pc-TUG1. (A) miR-17-5p expression was examined via reverse transcription quantitative PCR. (B) Cell viability was analyzed using a Cell counting kit 8 assay. (C) Cell apoptosis was assessed via flow cytometry analysis. (D) MMP13, collagen II and aggrecan protein levels were measured using western blotting. " $\mathrm{P}<0.05$ as indicated. TUG1, taurine upregulated gene 1; miR, microRNA; miR-NC, microRNA negative control; MMP13, matrix metalloprotein 13.

Tang et al (31) demonstrated that TUG1 was highly expressed in OA cartilage tissues and IL- $1 \beta$ or TNF- $\alpha$-stimulated chondrocytes, and that TUG1 promoted the degradation of chondrocyte ECM. In the present study, TUG1 knockdown promoted IL-1 $\beta$-induced chondrocyte viability and inhibited chondrocyte apoptosis and ECM damage.

TUG1 acted as a target for miR-17-5p in chondrocytes. miR-17-5p has been reported to be a key regulator in various human conditions, such as triple-negative breast cancer (32), hepatocellular carcinoma (33) and colorectal cancer (34). Furthermore, Hu et al (10) demonstrated that miR-17-5p was reduced in OA and its upregulation resulted in chondrocyte growth and the inhibition of chondrocyte apoptosis and ECM degradation in IL-1 $\beta$-induced OA (10). In the current study, miR-17-5p was weakly expressed in OA and its upregulation increased cell viability and decreased cell apoptosis and ECM degradation in IL-1 $\beta$-stimulated chondrocytes. However, the effect of miR-17-5p on OA progression was abolished by TUG1 overexpression.

Lai et al (35) demonstrated that FUT1 knockdown repressed cell growth and metastasis in breast cancer. Kawai et al (36) reported that FUT1 downregulation inhibited cell growth and 

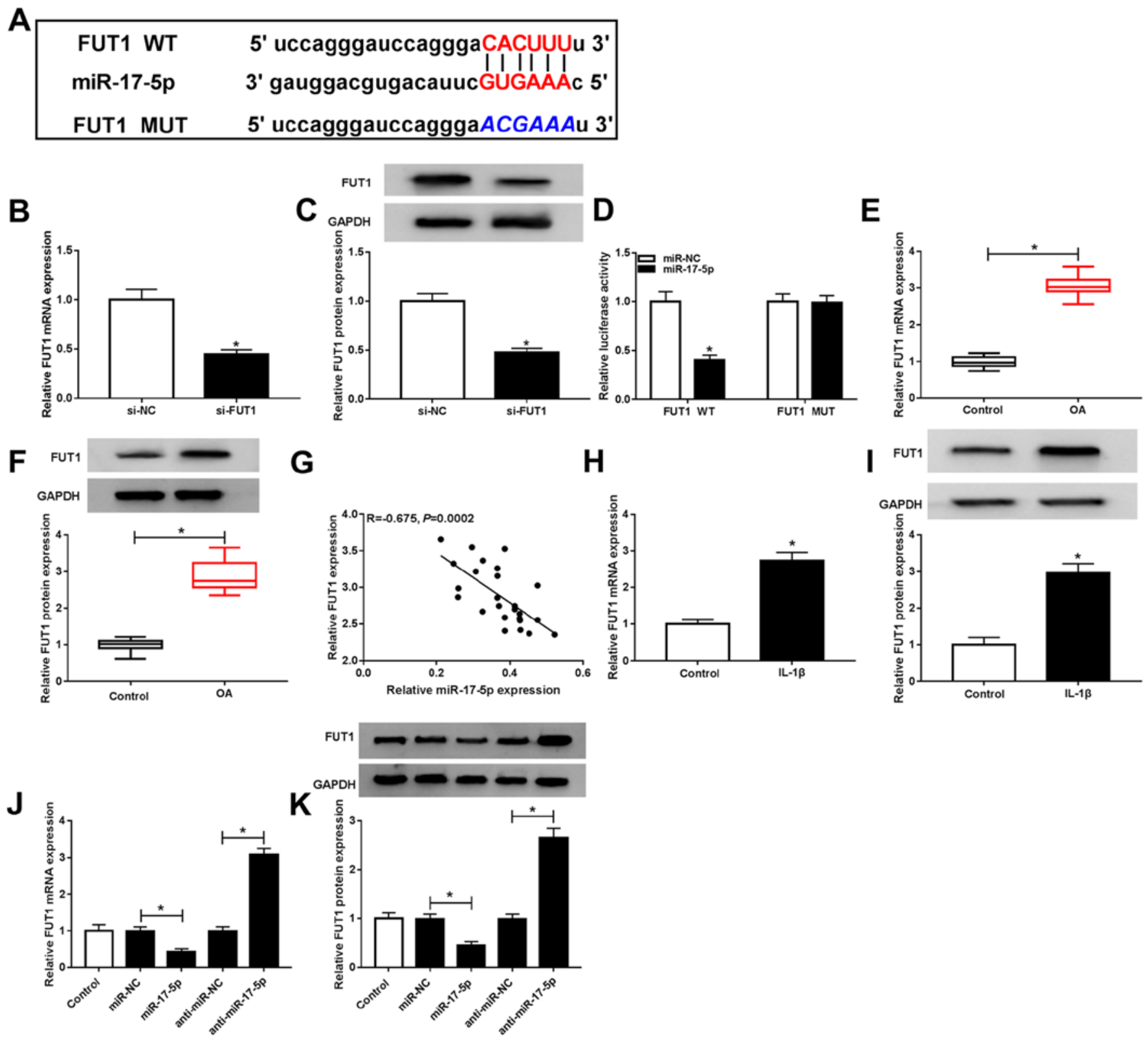

Figure 5. miR-17-5p inhibited FUT1 expression in chondrocytes. (A) Potential binding sites between miR-17-5p and FUT1 were predicted via starBase. si-NC or si-FUT1 was transfected into chondrocytes and FUT1 (B) mRNA and (C) protein expressions were determined via RT-qPCR and western blotting, respectively. (D) Luciferase activity between miR-17-5p and FUT1 was assessed using dual-luciferase reporter assay. FUT1 (E) mRNA and (F) protein levels in OA cartilage tissues and controls were analyzed by RT-qPCR and western blot analysis, respectively. (G) Correlation between miR-17-5p and FUT1 in OA cartilage tissues was determined via Spearman's correlation analysis. FUT1 (H) mRNA and (I) protein levels in IL-1 $\beta$-stimulated chondrocytes and controls were detected by RT-qPCR and western blotting, respectively. Chondrocytes were untransfected or transfected with miR-NC, miR-17-5p, anti-miR-NC or anti-miR-17-5p, after which FUT1 (J) mRNA and (K) protein expression was examined via RT-qPCR and western blotting, respectively. "P<0.05 vs. si-NC, "P<0.05 vs. miR-NC, "P<0.05 vs. Control. miR, microRNA; FUT1, fucosyltransferase 1; siRNA, small interfering RNA; si-NC, small interfering RNA negative control; si-FUT1, small interfering RNA targeting FUT1; RT-qPCR, reverse transcription quantitative-PCR; OA, osteoarthritis; IL, interleukin; miR-NC, microRNA negative control; anti-miR-NC, anti-microRNA negative control; anti-miR-17-5p, mir-17-5p inhibitor.

promoted cell apoptosis in NCL-N87 cells. These results indicated that FUT1 was involved in the development of human disease. In the present study the function of FUT1 in OA was investigated. FUT1 served as a target for miR-17-5p in chondrocytes and there was an inverse correlation between FUT1 and miR-17-5p. Furthermore, the effect of FUT1 knockdown on cell viability, apoptosis and ECM degradation were partly reversed by the inhibition of miR-17-5p in IL-1 $\beta$-activated chondrocytes.
In conclusion, TUG1 knockdown decelerated OA progression by promoting chondrocyte viability and repressing chondrocyte apoptosis and cartilage ECM damage. Furthermore, TUG1 modulated OA progression via the miR-17-5p/FUT1 pathway. These results may further elucidate OA pathogenesis and may provide a method of effective treatment for patients with OA. However, the current study had limitations, such as an insufficient sample size. In addition, the IL- $1 \beta$-induced chondrocytes used to simulate OA condition 
A

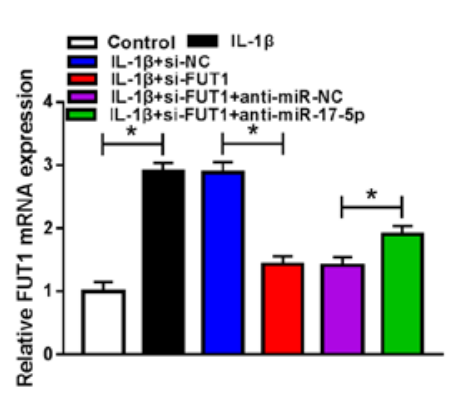

FUT1

B $\mathrm{GAPDH}$

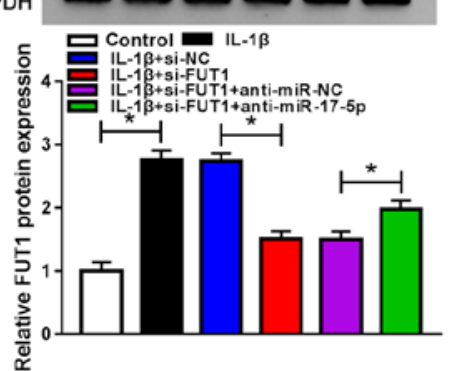

C

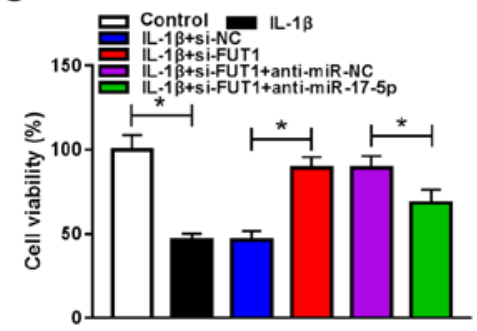

D
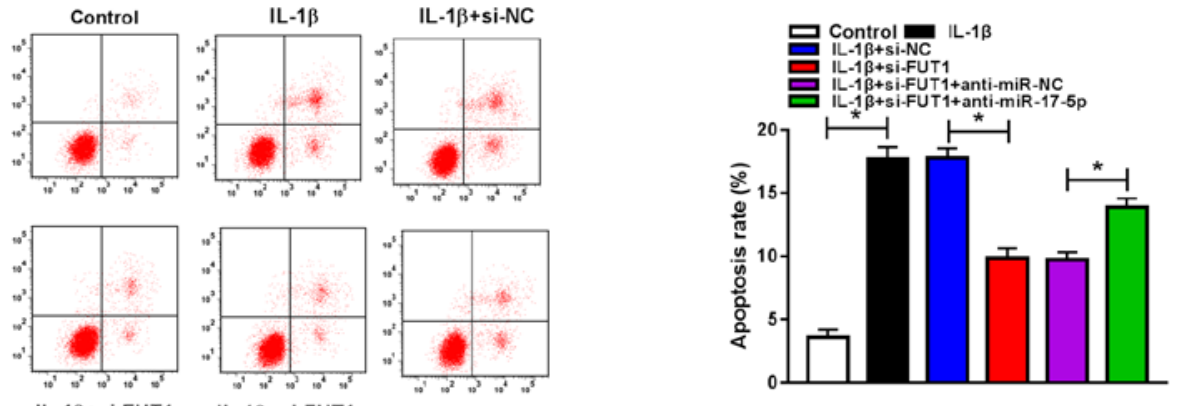

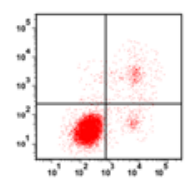

IL-1 $\beta+$ si-FUT1

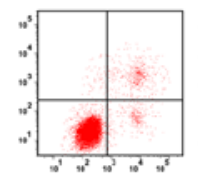

IL-1 $\beta+$ si-FUT1+ anti-miR-NC
IL-1 $\beta+$ si-NC
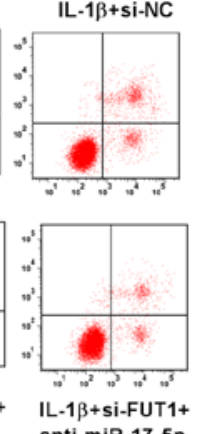

anti-miR-17-5p

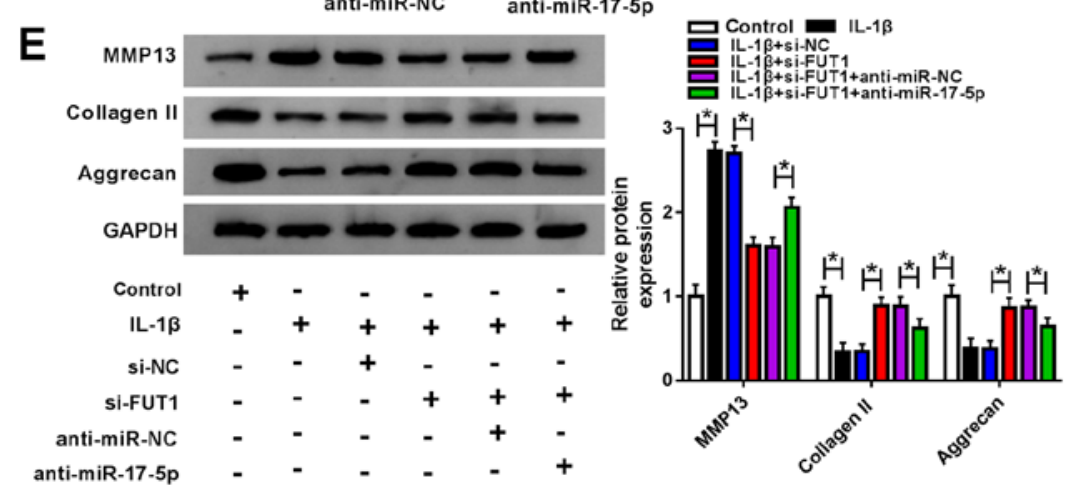

Figure 6. miR-17-5p regulated cell viability, apoptosis and extracellular matrix degradation by targeting FUT1 in IL-1 $\beta$-induced chondrocytes. Chondrocytes were untransfected or transfected with IL-1 $\beta$, IL-1 $\beta+$ si-NC, IL-1 $\beta+$ si-FUT1, IL-1 $\beta+$ si-FUT1 + anti-miR-NC or IL-1 $\beta+$ si-FUT1 + anti-miR-17-5p. (A) FUT1 mRNA expression was measured via reverse transcription quantitative PCR. (B) FUT 1 protein expression was examined using western blotting. (C) Chondrocyte viability was assessed via a Cell Counting kit-8 assay. (D) Chondrocyte apoptosis was evaluated through flow cytometry analysis. (E) MMP13, collagen II and aggrecan protein levels were analyzed using western blotting. " $\mathrm{P}<0.05$ as indicated. miR, microRNA; FUT1, fucosyltransferase 1; IL, interleukin; siRNA, small interfering RNA; si-NC, small interfering RNA negative control; si-FUT1, small interfering RNA targeting FUT1; anti-miR-NC, anti-microRNA negative control; anti-miR-17-5p, miR-17-5p inhibitor; MMP13, matrix metalloprotein 13.
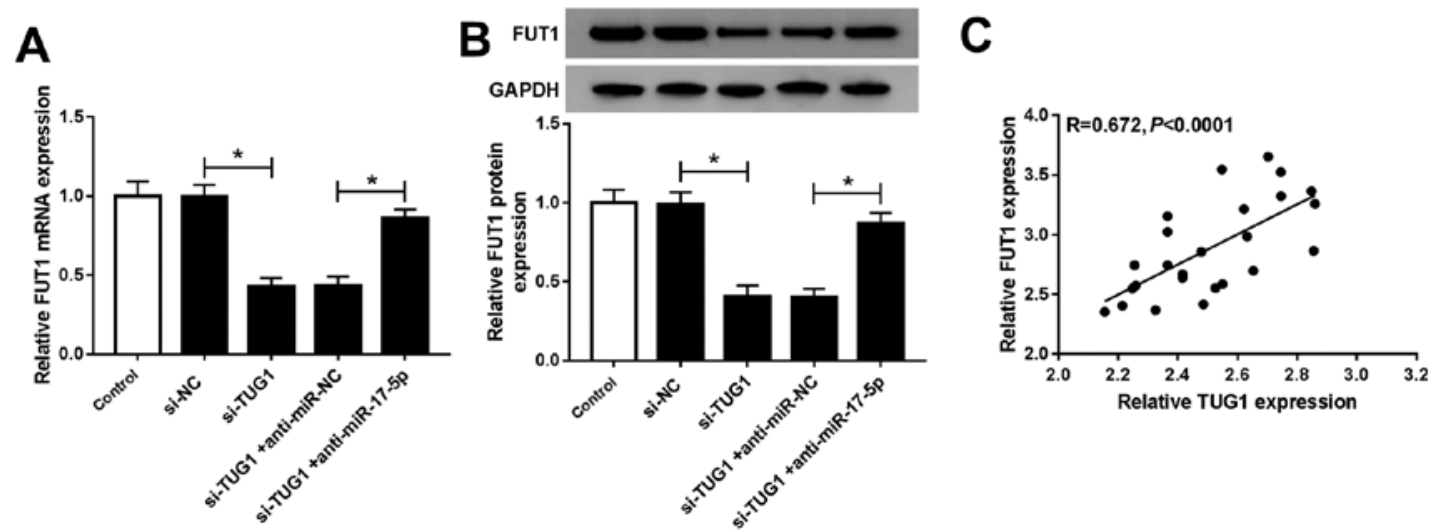

Figure 7. TUG1 upregulated FUT1 expression by targeting miR-17-5p in chondrocytes. FUT1 (A) mRNA and (B) protein levels in chondrocytes untransfected or transfected with si-NC, si-TUG1, si-TUG1 + anti-miR-NC or si-TUG1 + anti-miR-17-5p were determined using reverse transcription quantitative PCR and western blotting, respectively. (C) Spearman's correlation analysis was used to determine the association between FUT1 mRNA and TUG1. "P<0.05 as indicated. TUG1, taurine upregulated gene 1; miR, microRNA; siRNA, small interfering RNA; si-NC, small interfering RNA negative control; si-TUG1, small interfering RNA targeting TUG1; anti-miR-NC, anti-microRNA negative control; anti-miR-17-5p, mir-17-5p inhibitor; FUT1, fucosyltransferase 1. 
may have limitations, we did not exclude additional mechanisms such as how TUG1 might affect IL-1 $\beta$. The role of the TUG/miR-17-5p/FUT1 pathway in other processes involved in OA development requires further investigation.

\section{Acknowledgements}

Not applicable.

\section{Funding}

No funding was received.

\section{Availability of data and materials}

The datasets used and/or analyzed during the current study are available from the corresponding author on reasonable request.

\section{Authors' contributions}

ZL and JY designed the current study. ZL drafted the manuscript. JW revised the manuscript for important intellectual content and performed statistical analysis. All authors read and approved the final manuscript and agreed to be held accountable for all aspects of the current work in ensuring that questions related to the accuracy or integrity of any part of the current work are appropriately investigated and resolved.

\section{Ethics approval and consent to participate}

The current study was approved by the Ethics Committee of Hanyang Hospital affiliated to Wuhan University of Science and Technology and patients provided written informed consent.

\section{Patient consent for publication}

Not applicable.

\section{Competing interests}

The authors declare that they have no competing interests.

\section{References}

1. Arden N and Nevitt MC: Osteoarthritis: Epidemiology. Best Pract Res Clin Rheumatol 20: 3-25, 2006.

2. Michael JW, Schlüter-Brust KU and Eysel P: The epidemiology, etiology, diagnosis, and treatment of osteoarthritis of the knee. Dtsch Arztebl Int 107: 152-162, 2010.

3. Rahmati M, Nalesso G, Mobasheri A and Mozafari M: Aging and osteoarthritis: Central role of the extracellular matrix. Ageing Res Rev 40: 20-30, 2017.

4. Van der Kraan P, Buma P, Van Kuppevelt T and Van Den Berg W: Interaction of chondrocytes, extracellular matrix and growth factors: Relevance for articular cartilage tissue engineering. Osteoarthritis Cartilage 10: 631-637, 2002.

5. Cross M, Smith E, Hoy D, Nolte S, Ackerman I, Fransen M, Bridgett L, Williams S, Guillemin F, Hill CL, et al: The global burden of hip and knee osteoarthritis: Estimates from the global burden of disease 2010 study. Ann Rheum Dis 73: 1323-1330, 2014

6. Johnsson P, Lipovich L, Grandér D and Morris KV: Evolutionary conservation of long non-coding RNAs; sequence, structure, function. Biochim Biophys Acta 1840: 1063-1071, 2014.
7. Mercer TR, Dinger ME and Mattick JS: Long non-coding RNAs: Insights into functions. Nat Rev Genet 10: 155-159, 2009.

8. Niu Y, Ma F, Huang W, Fang S, Li M, Wei T and Guo L: Long non-coding RNA TUG1 is involved in cell growth and chemoresistance of small cell lung cancer by regulating LIMK $2 \mathrm{~b}$ via EZH2. Mol Cancer 16: 5, 2017.

9. Huang MD, Chen WM, Qi FZ, Sun M, Xu TP, Ma P and Shu YQ: Long non-coding RNA TUG1 is up-regulated in hepatocellular carcinoma and promotes cell growth and apoptosis by epigenetically silencing of KLF2. Mol Cancer 14: 165, 2015.

10. Hu J, Wang Z, Shan Y, Pan Y, Ma J and Jia L: Long non-coding RNA HOTAIR promotes osteoarthritis progression via miR-17-5p/FUT2/ $\beta$-catenin axis. Cell Death Dis 9: 711, 2018.

11. Chen K, Zhu H, Zheng $\mathrm{M}-\mathrm{Q}$ and Dong QR: lncRNA MEG3 inhibits the degradation of the extracellular matrix of chondrocytes in osteoarthritis via targeting miR-93/TGFBR2 Axis. Cartilage: Jun 28, 2019 (Epub ahead of print). doi: $10.1177 / 1947603519855759$.

12. Li L, Lv G, Wang B and Kuang L: The role of lncRNA XIST/miR-211 axis in modulating the proliferation and apoptosis of osteoarthritis chondrocytes through CXCR4 and MAPK signaling. Biochem Biophys Res Commun 503: 2555-2562, 2018.

13. He L and Hannon GJ: MicroRNAs: Small RNAs with a big role in gene regulation. Nat Rev Genet 5: 522-531, 2004.

14. Huang J, Zhao L, Fan Y, Liao L, Ma PX, Xiao G and Chen D: The microRNAs miR-204 and miR-211 maintain joint homeostasis and protect against osteoarthritis progression. Nat Commun 10: $2876,2019$.

15. Meng F, Li Z, Zhang Z, Yang Z, Kang Y, Zhao X, Long D, $\mathrm{Hu} \mathrm{S}, \mathrm{Gu} \mathrm{M}, \mathrm{He} \mathrm{S}$, et al: MicroRNA-193b-3p regulates chondrogenesis and chondrocyte metabolism by targeting HDAC3. Theranostics 8: 2862-2883, 2018.

16. Cai C,Min S, Yan B,Liu W, Yang X,LiL, Wang T and Jin A: miR-27a promotes the autophagy and apoptosis of IL-1 $\beta$ treated-articular chondrocytes in osteoarthritis through $\mathrm{PI} 3 \mathrm{~K} / \mathrm{AKT} / \mathrm{mTOR}$ signaling. Aging (Albany NY) 11: 6371-6384, 2019.

17. Yang B, Kang X, Xing Y, Dou C, Kang F, Li J, Quan Y and Dong S: Effect of microRNA-145 on IL-1 $\beta$-induced cartilage degradation in human chondrocytes. FEBS Lett 588: 2344-2352, 2014.

18. Pilyugin $M$ and Irminger-Finger I: Long non-coding RNA and microRNAs might act in regulating the expression of BARD1 mRNAs. Int J Biochem Cell Biol 54: 356-367, 2014.

19. Mizuochi T, Taniguchi T, Shimizu A and Kobata A: Structural and numerical variations of the carbohydrate moiety of immunoglobulin G. J Immunol 129: 2016-2020, 1982.

20. Hu J, Wang Z, Pan Y, Ma J, Miao X, Qi X, Zhou H and Jia L: miR-26a and miR-26b mediate osteoarthritis progression by targeting FUT4 via NF- $\kappa$ B signaling pathway. Int J Biochem Cell Biol 94: 79-88, 2018.

21. Ma B, Simala-Grant JL and Taylor DE: Fucosylation in prokaryotes and eukaryotes. Glycobiology 16: 158R-184R, 2006.

22. Livak KJ and Schmittgen TD: Analysis of relative gene expression data using real-time quantitative PCR and the 2(-Delta Delta C(T)) method. Methods 25: 402-408, 2001.

23. Lu J, Deng ZH, Li YS and Lei GH: Long noncoding RNAs in osteoarthritis. Joint Bone Spine 84: 553-556, 2017.

24. Xing D, Liang Jq, Li Y, Lu J, Jia Hb, Xu Ly and Ma Xl: Identification of long noncoding RNA associated with osteoarthritis in humans. Orthop Surg 6: 288-293, 2014.

25. Aida Y, Maeno M, Suzuki N, Shiratsuchi H, Motohashi M and Matsumura $\mathrm{H}$ : The effect of IL-1beta on the expression of matrix metalloproteinases and tissue inhibitors of matrix metalloproteinases in human chondrocytes. Life Sci 77: 3210-3221, 2005.

26. Daheshia M and Yao JQ: The interleukin lbeta pathway in the pathogenesis of osteoarthritis. J Rheumatol 35: 2306-2312, 2008

27. Lopez-Armada MJ, Carames B, Lires-Dean M, Cillero-Pastor B, Ruiz-Romero C, Galdo F and Blanco FJ: Cytokines, tumor necrosis factor-alpha and interleukin-1beta, differentially regulate apoptosis in osteoarthritis cultured human chondrocytes. Osteoarthritis Cartilage 14: 660-669, 2006.

28. Takahashi A, de Andrés MC, Hashimoto K, Itoi E, Otero M, Goldring MB and Oreffo ROC: DNA methylation of the RUNX2 P1 promoter mediates MMP13 transcription in chondrocytes. Sci Rep 7: 7771, 2017.

29. Larkin J, Lohr TA, Elefante L, Shearin J, Matico R, Su JL, Xue Y, Liu F, Genell C, Miller RE, et al: Translational development of an ADAMTS-5 antibody for osteoarthritis disease modification. Osteoarthritis Cartilage 23: 1254-1266, 2015. 
30. Liang Z and Ren C: Emodin attenuates apoptosis and inflammation induced by LPS through up-regulating lncRNA TUG1 in murine chondrogenic ATDC5 cells. Biomed Pharmacothery 103 : 897-902, 2018

31. Tang LP, Ding JB, Liu ZH and Zhou GJ: IncRNA TUG1 promotes osteoarthritis-induced degradation of chondrocyte extracellular matrix via miR-195/MMP-13 axis. Euro Rev Med Pharmacol Sci 22: 8574-8581, 2018.

32. Li J, Lai Y, Ma J, Liu Y, Bi J, Zhang L, Chen L, Yao C, Lv W, Chang G, et al: miR-17-5p suppresses cell proliferation and invasion by targeting ETV1 in triple-negative breast cancer. BMC Cancer 17: 745, 2017.

33. Yang F, Yin Y, Wang F, Wang Y, Zhang L, Tang Y and Sun S: miR-17-5p Promotes migration of human hepatocellular carcinoma cells through the $\mathrm{p} 38$ mitogen-activated protein kinase-heat shock protein 27 pathway. Hepatology 51: 1614-1623, 2010.
34. Ma Y, Zhang P, Wang F, Zhang H, Yang Y, Shi C, Xia Y, Peng J, Liu W, Yang Z and Qin H: Elevated oncofoetal miR-17-5p expression regulates colorectal cancer progression by repressing its target gene P130. Nat Commun 3: 1291, 2012.

35. Lai TY, Chen IJ, Lin RJ, Liao GS, Yeo HL, Ho CL, Wu JC, Chang NC, Lee AC and Alice LY: Fucosyltransferase 1 and 2 play pivotal roles in breast cancer cells. Cell Death Discov 5: 74, 2019.

36. Kawai S, Kato S, Imai H, Okada Y and Ishioka C: Suppression of FUT1 attenuates cell proliferation in the HER2-overexpressing cancer cell line NCI-N87. Oncol Rep 29: 13-20, 2013.

(i) () $\odot$ This work is licensed under a Creative Commons No At Atribution-NonCommercial-NoDerivatives 4.0 International (CC BY-NC-ND 4.0) License. 